\title{
Clinicians' Perspectives About the Feasibility and Effectiveness of Using Telemedicine in First Aid: A Qualitative Study
}

\author{
Xiaoqun Fang' \\ Shulei Jia ${ }^{2}$ \\ Qiuyan Wang' \\ Hui-fang Liu' \\ Yumei Zhou' \\ Lingling Zhang' \\ Tanghua Dai' \\ Hui Luo' \\ Hui Peng' \\ Jun Yuan ${ }^{2}$ \\ Huyan Zhou ${ }^{2}$ \\ 'Department of Rehabilitation Medicine, \\ The Second Affiliated Hospital of \\ Nanchang University, Nanchang, 330006, \\ People's Republic of China; ${ }^{2}$ School of \\ Nursing, Nanchang University, Nanchang, \\ 330006, People's Republic of China
}

Correspondence: Shulei Jia

School of Nursing, Nanchang University, No. 46I of Ba Yi Road, Donghu District, Nanchang, 330006, People's Republic of China

Tel $+86 I 579786 \quad 1504$

Fax +8679186360530

Email jiashulei202I@163.com
Objective: This study investigated clinicians' perspectives on the feasibility and effectiveness of using telemedicine in the context of providing first aid. It is crucial to identify and explore clinicians' attitudes and awareness of tele-first-aid in China to keep pace with ongoing global trends.

Design: This was a qualitative study. Data gathered from in-depth interviews with 22 clinicians were coded into themes and analyzed.

Participants: Participants included hospital-based clinicians: four clinical specialists, eight emergency nurses, four emergency doctors, three general practitioners, and three clinical administrators.

Setting: The study was conducted in university-affiliated hospitals.

Results: All clinicians who were invited to participate believed that the use of telemedicine in first aid was promising and effective. Participants stated that relevant technology should be designed to synergize the chain of existing platforms in the industry and that it should be determined who has the authority to utilize such technology and how it should be used to achieve the sought-after benefits. The use of such technology refreshes the role of clinicians and their relationship with patients. Additionally, participants believed that the government could play an important role especially in the reform of medical systems.

Conclusion: Telemedicine can facilitate the provision of conventional first aid and systematically exploit existing resources due to its feasibility and effectiveness. The social benefits of using tele-first-aid in clinical and community applications, especially when used in coordination with existing resources, make its adoption an inevitable trend in the field.

Keywords: telemedicine, first aid, qualitative study, clinicians

\section{Introduction}

In China, over 1.8 million people die in accidents every year. ${ }^{1}$ Most of them fail to receive first aid promptly, which increases the likelihood of death. ${ }^{2,3}$ Treatment delay can be a factor leading to aggravated complications that can result in death. ${ }^{4}$ Moreover, in most underdeveloped, remote cities in China, there is also a critical shortage of first aid professionals. Providing urgent healthcare following certain accidents can, therefore, be challenging, and telemedicine could be an option in such cases.

Telemedicine uses forms of telecommunication such as computers and internet technology to provide specialist medical services to other medical institutions or directly to patients. This system can utilize medical resources efficiently and solve 
problems resulting from the "difficulties in medical care" in China. ${ }^{5}$ Tele-first-aid is one branch of telemedicine. It operates using remote networks and real-time monitors that connect the emergency departments of hospitals with ambulances and callers. ${ }^{6,7}$ In addition, through an intelligent image classification module and wireless transmission devices, all relevant data, including patient history data, can be transmitted to the expert guidance center and recorded automatically. ${ }^{8}$ The system will select the fastest route and send this data to the ambulance to direct it to the accident location, shortening the time it takes medical workers to find the injured person. ${ }^{9-12}$ Such systems can also learn from the process of diagnosis and the practitioners' advice, which help the systems become a self-improving loop..$^{9,13-15}$ Tele-first-aid has become increasingly popular in emergency departments and China, and tele-first-aid services are offered by 120 ambulance systems. There has also been an associated increase in published research on this subject. ${ }^{16-18}$ However, tele-first-aid is still not popular in most areas of China, and there has been little scrutiny of the clinicians providing tele-first-aid work, so little is known about their endeavors. Most of the previous literature explored the development and application of remote first aid systems but ignored the attitudes and ideas of the users of these systems. Therefore, from the perspective of potential remote first aid system users, this paper explores their attitudes, opinions, and suggestions toward this system. The extensive discussion is helpful to optimize the remote emergency system. These can be further optimized not only from the designer's point of view but also from the user's point of view. In addition, it helps deepen the public's understanding of the remote emergency system and lays the foundation for the popularization of the system.

This study aimed to explore the feasibility and effectiveness of tele-first-aid and provide a reference for developing and applying tele-first-aid in cities. We recruited clinical specialists, emergency nurses and doctors, general practitioners, and clinical administrators and conducted indepth interviews on their opinions of telemedicine technology and its use in practice. The findings from the in-depth interviews are reported as follows.

\section{Methods}

\section{Study Design}

First, qualitative in-depth interviews were conducted with hospital-based clinicians. Then, thematic analysis, one of the most common forms of analysis in qualitative research, was used to process the collected data. The most widely cited approach to thematic analysis is one developed by psychologists Virginia Braun and Victoria Clarke. ${ }^{13-15}$ Their approach involves six phases of coding to establish meaningful themes. In this study, all researchers had prior experience with qualitative studies and had trained specifically for the project. The clinical participants provided written informed consent prior to the interview.

\section{Participants}

We recruited 22 clinicians (aged 30-54) working in firstclass hospitals in Henan and Jiangxi provinces, where basic hospitals have been found to provide lower-quality medical services than hospitals in developed cities. Between June and December 2019, clinicians were invited by telephone to join the project.

First, all 22 participants participated in and passed the training program of the remote emergency system in October 2018 and April 2019. The training content includes the overview, background, technology, function, prospects, and limitations of the remote first aid system. The 22 participants were familiar with remote emergency systems. Second, all 22 participants visited and tried out the remote emergency system of a company in Shanghai. They all had a perceptual and rational understanding of the remote first aid system. Third, 25 people were randomly selected from 326 people who were trained in the remote first aid system and visited the site. Three people were unable to participate in the study for various reasons, and 22 people volunteered to participate. These 22 clinicians were all involved in prehospital medical care, ${ }^{16}$ including four clinical specialists, eight emergency room nurses, four emergency room doctors, three general practitioners, and three clinical administrators. Table 1 provides the participants' demographics, including their gender, age range, and employment tenure.

\section{Interviews}

We conducted semi-structured interviews (Figure 1), asking clinicians relevant, open-ended questions to explore their preferences regarding tele-first-aid. Each interview lasted between 30 and 45 minutes, was recorded, and then transcribed verbatim. The questions were asked one by one and covered issues related to prehospital tele-first-aid usage, its impact on emergency outcomes, and so forth. Data analysis was conducted during the interviews in the form of circulation. Interviews were ceased once information saturation 
Table I Descriptive Characteristics of the Study Participants

\begin{tabular}{|c|c|c|c|c|c|}
\hline Age(Years) & Gender & $\begin{array}{l}\text { Participants } \\
\text { Number }\end{array}$ & $\begin{array}{l}\text { Employment } \\
\text { Period (Range) }\end{array}$ & $\begin{array}{l}\text { Prehospital Medical } \\
\text { Employment Period (Range) }\end{array}$ & $\begin{array}{l}\text { Training Experience of } \\
\text { Telemedicine in First Aid }\end{array}$ \\
\hline $30-40$ & $\begin{array}{l}M \\
F\end{array}$ & $\begin{array}{l}4 \\
7\end{array}$ & $\begin{array}{l}{[5-13]} \\
{[7-14]}\end{array}$ & $\begin{array}{l}{[4-10]} \\
{[4-12]}\end{array}$ & $\begin{array}{l}Y \\
Y\end{array}$ \\
\hline $4 I-50$ & $\begin{array}{l}M \\
F\end{array}$ & $\begin{array}{l}2 \\
6\end{array}$ & $\begin{array}{l}{[16-27]} \\
{[15-28]}\end{array}$ & $\begin{array}{l}{[5-23]} \\
{[6-28]}\end{array}$ & $\begin{array}{l}Y \\
Y\end{array}$ \\
\hline$>50$ & $\begin{array}{l}M \\
F\end{array}$ & $\begin{array}{l}1 \\
2\end{array}$ & $\begin{array}{l}{[30]} \\
{[29-36]}\end{array}$ & $\begin{array}{l}{[23]} \\
{[27-30]}\end{array}$ & $\begin{array}{l}Y \\
Y\end{array}$ \\
\hline Total & & 22 & & & \\
\hline
\end{tabular}

Abbreviations: $M$, male; F, female; Y, yes; $N$, no.

was achieved, although several additional interviews were conducted to confirm saturation.

\section{Qualitative Data Analysis}

A process of thematic analysis was used to analyze the transcribed information from the interviews. We followed several steps to capture the views of the clinicians: familiarizing ourselves with the data, generating the initial codes, establishing preliminary themes among the codes, reviewing and reanalyzing themes, defining and clarifying the key themes, and producing the final results.

\section{Results}

Clinicians shared their general knowledge and personal experiences of telemedicine. Telemedicine in first aid was regarded as a system to exchange information and support to both patients and junior clinicians through reassurance and guidance. Four main themes were identified: utility, clinician-patient relationship, cooperation of organizations, and barriers to practice. In the following quotes, CS indicates a clinical specialist, EN indicates an emergency nurse, ED indicates an emergency doctor, GP indicates a general practitioner, and $\mathrm{CA}$ indicates a clinical administrator.

\section{Utility}

All participants agreed that telemedicine technology could be used clinically. The main applications of the technology are summarized in Table 2 .

Clinicians conveyed that even advanced telemedicine could not replace their expertise but could facilitate in confirming most patients' condition and accurately analyzing the opinions of clinicians during ambulance rides or prior to the arrival of professionals:

"I am not worried about being replaced, because the experience, the details, psychological changes ... machines cannot handle such complicated situations." (EN1)

Traditional face-to-face assessment will remain the mainstay, especially for internal and sudden illnesses. In some cases, it has been assumed that the client's condition is too complicated for a definitive diagnosis to be made or firstaid to be provided using telemedicine. However, clinicians agreed that telemedicine data could be used for reference:

"We cannot tell how serious the damage to brain or bone is just through video. The appearance of a real-time situation, or descriptions from witnesses, you know, may be wrong." (ED2)

"I don't think all emergency measures are suitable for remote first aid, such as patients who need a tracheal cannula immediately. But those who have heavy bleeding or heart failure may be saved by FaceTime guidance." (CS2)

\section{Patient Data Sharing and Treatment Plan Design}

Telemedicine was recognized as being useful for gathering objective human data from a distance, exchanging subjective opinions on clinical experience, and supporting evidence-based decision-making. The clinicians agreed that it is useful to see the patient on FaceTime with their own eyes to have a clearer understanding of the patient's current condition:

"We can see the patient face-to-face through the wireless display and can give some guidance to them while they're 


\section{Contextual background}

1. Describe your professional job.

2. What types of patients do you contact with?

3. Does most of your work involve following an ambulance to rescue victims?

4. What is the best part in your work? What is the worst part?

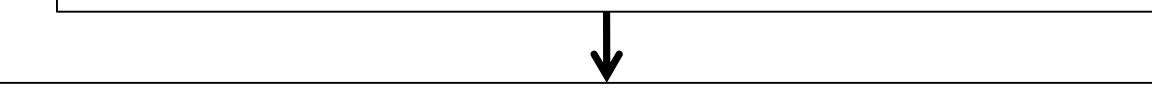

\section{Prehospital tele-first- aid}

1. Do you know anything about the prehospital tele-first- aid?

2. If so, tell me what you know.

3. Does your department carry out the prehospital tele-first- aid work?

4. From what you know, what's your view of the prehospital tele-first- aid?

5. How would you use them? How do you carry out the tele-first- aid work?

A. Is the prehospital tele-first-aid beneficial for patients? For What benefits?

B. Is the prehospital tele-first-aid beneficial for clinicians? For What benefits?

6. What information do you most want to receive before emergency patients come to the hospital?

7. Would you use the data in the tele-first-aid? Why or why not?

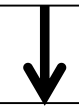

Impact of the telemedicine on first-aid or rescue in hospital

1. What do you think of the inadequacy and weakness for the tele-first-aid?

2. What do you think of the effect for succeeding rescue work in hospital?

Closure
Is there anything else that you would like to add about the tele-first- aid that
we haven't covered here?

Figure I Open-ended interview questions. 
Table 2 Themes and Subthemes Determined from the Results

\begin{tabular}{|l|l|l|}
\hline Theme & Subtheme & The Numbers of Participants' Responses \\
\hline Utility & Support treatment planning & 19 \\
& Efficient management & 18 \\
& Data and resource sharing & 21 \\
& Mental support & 15 \\
& Better working environment & 19 \\
\hline Clinician-patient relationship & Enhanced by high quality services & 16 \\
\hline Cooperation of organizations & Government supported & 18 \\
& Medical treatment combination & 20 \\
\hline Barriers on practice & Public including users Protected by law & 21 \\
\hline & Learning process and time & 17 \\
& Cost and technology & 20 \\
& Public's understand and acceptance & 19 \\
& Relative law & 16 \\
\hline
\end{tabular}

waiting for the ambulance. It is more intuitive and precise than giving an oral description by phone." (ED4)

Many experts and technical staff described telemedicine as a system that can collect not only clients' vital signs and biochemical markers from wireless devices but also their identity information and medical history from a data center. These data are helpful for clinicians in diagnosing and caring for patients:

"Big data platforms, we always say this buzzword in China, can gather information on patient history, vital signs, specialist advice, and similar cases to create superior treatment plans. I think it is good to have this history file to support care." (EN7)

When patients are sent to the hospital, they may not be able to obtain the best treatment plan if clinician resources or medical devices are limited. However, tele-first-aid can improve this by connecting such hospitals with superior hospitals:

"I find it difficult to solve some problems ... but if we could contact a superior hospital through this system ... I mean, it is amazing to get better guidance and more care from a larger hospital, you know, with rich resources in terms of scientific research and experts as well.” (GP2)

Wider case collection and the maximization of clinical resources were noted as helping to meet patients' needs:

"It will promote a more rational distribution of medical resources among different levels of hospitals. I mean, the intelligence system can match patients' needs." (CS3)

\section{Prehospital First-Aid Participation and Measures}

The core function of tele-first-aid was identified as realtime emergency management. The clinicians noted that if a person has an accident far from the hospital, they can ask for help through a telemedicine app. The receiving terminal of the telemedicine system can receive the request and action it while simultaneously processing the information and sending it to relevant clinicians. Passers-by can also lend a hand if they come across a case that requires first aid:

"If the first eyewitness can join the emergency effort and successfully get instructions from professionals, everyone in our society would be motivated and want to learn some first-aid skills." (EN8)

Information on the first-aid knowledge and skills of laypeople emerged from the interviews. It is clear that the role of laypeople in first aid is essential, and technical education in an audio-visual form can motivate laypeople to learn how to provide effective treatment:

"It is possible to communicate online, for example, if a person is seriously cut or bitten outside. He or she can get instructions from the system to press on the upper vessel to stop the bleeding until the doctors arrive." (ED3)

\section{Managing Referral and Latency Time}

Using this technology, GPS can quickly and accurately determine the location of the patient. Through the video- 
audio system, more detailed information about the situation can be obtained. The ambulance can be guided to find the fastest route to the patient's location, which can save valuable time:

"We can get to the location easily and speak anytime. It will save time." (EN3)

Meanwhile, through communication, preparations can be made in advance, and time can be saved during admission and referral:

"While the patient is on the way to the hospital, surgical and treatment equipment is prepared in advance based on the patient's condition. Or, if we can consult professionals from other hospitals early and implement the referral as soon as possible, this would undoubtedly be fantastic." (EN4)

\section{Clinician-Patient Relationship}

By and large, all clinicians perceived that the clinicianpatient relationship could be enhanced by high-quality services:

"I think the patients will be more confident in doctors because they can get professional help while the ambulance is on its way. Without this technology, the patient may be alone." (EN5)

The confidence provided by doctors can comfort and reassure patients, who may otherwise feel afraid or anxious when tragedy or an accident occurs:

"Patients usually feel anxious when facing accidents; doctors on the video screen can calm them." (EN2)

Clinicians and patients can exchange a great deal of information through the system and can help them get to know each other better, thus preventing the formation of negative public opinion:

"Chinese patients have high expectations of doctors, wanting them to cure diseases quickly and completely. But you know, we're not God, and it's far beyond our ability sometimes. Telemedicine, I think, to some degree, is more objective and just, helping us avoid negative public opinion and resulting in a full mutual understanding." (ED1)

This system can improve the working atmosphere for medical staff. For example, allowing the participation of laypeople in the medical field and letting them experience the hardships experienced by professional medical staff may enhance the mutual understanding between the two groups:

"[Telemedicine can allow] the public to experience our actual situation and reactions. It's also a method to eliminate their worries and distrust because we hold the common aim of recovering from illness in that case." (EN2)

\section{Cooperation of Organizations}

In terms of the practicality of prehospital telemedicine, the clinicians suggested that tele-first-aid should be involved in multiple areas and be government-supported:

"Who administrates the data? Who has the authority to access the database? I mean, to use it?" (CS4)

"We need to build up the tele-first-aid interface and operating system. And at the same time, it should have rules and standards for third parties to represent clinicians and manage the real-time digital files." (CA1)

Hospitals should have a detailed and definitive ranking system that is optimally integrated so that it is convenient to transfer patients to superior hospitals if necessary. Professional clinical teams should also be trained in telemedicine:

"I think an exclusive office of telemedicine should be taken into consideration accordingly, and we should be trained [on it]." (GP3)

"There should be special educators to teach both the public and clinicians how to use the system. For instance, how to communicate the patient's location and condition, and how to use the camera." (CS1)

"It depends. Minor burns can be tele-tutored by community clinics, while complex conditions can be managed by big hospitals." (GP1)

Some participants also pointed out how the system can be used in certain specific situations where responsibility for keeping tele-first-aid safe and effective is unclear:

"If the patient passes out and others can't open her mobile phone, how can we get her general data and history?" (EN4)

In terms of emergency treatment responsibilities, the participants emphasized that there may be some difficulties during the consultation process, and some laypeople would prefer to protect themselves and leave rather than try to 
help people without hesitation, under the doctor's guidance:

"If the patient is severely allergic to the medicine the telemedicine system suggests, it's a huge deal." (ED2)

"I mean, if I consult professionals from another hospital, and then a medical accident occurs, say, hmm, the patient dies, under their instruction, who should take responsibility for that? Further, they may ask, why did you ask other hospitals for advice since you've got your own trained nurses? It's awkward." (EN4)

\section{Barriers to Practice}

The greatest concern identified during the interviews was the difficulty of understanding and interpreting the telemedicine technology:

"When we learned new technology, it took a lot of time and even occupied my spare time. I feel that learning new technology is very stressful.” (EN5)

"In China, the medical workload is extremely heavy, and I'm not interested in providing emergency services through a network. It's not realistic for me." (EN6)

Cost-effectiveness and internet technology were also described as barriers:

"I am not sure how far the technology goes today and whether the cost is affordable for the health department." (CA4)

"The speed of the network must be very fast and steady. Otherwise, the voice and image will be interrupted." (GP1)

However, these barriers can be overcome:

"If the benefits and feasibility could be supported by the government, it would be much easier [to implement] in China." (CA3)

"I wonder whether this idea can be supported by leaders, and what about taking the medical staff's workload into consideration or adding rewards for applying the new system?" (ED3)

Popular acceptance should be taken into account, according to the participants interviewed:

"Compliance is really an issue, as I wonder if patients will actually wear these devices (part of the system used to supervise patients and send alerts) at home." (EN3)
"The most important thing is the participation of the public and clinicians. I mean, if there are no professional responders online, it's useless." (EN2)

In addition, small-scale tests are necessary to examine and improve the attitude of the public toward tele-first-aid:

"People should be selected to try the first-aid app, and people with higher education levels are more likely to accept the app and cooperate. It's better to implement [tele-first-aid] in one area so that patients can be saved in a timely manner." (CS2)

\section{Discussion}

In this study, using in-depth interviews, we investigated and analyzed the clinicians' views on the feasibility and effectiveness of using telemedicine in first aid to understand the awareness and use of this technology. From the literature, we identified only a few studies that have investigated clinicians' perceptions of tele-first-aid. ${ }^{6,19}$ Most studies have concentrated on patient experiences, expectations, and recommendations for telemedicine design. Rarely were the views of clinicians in the specific field of first aid or the role of the general population examined. ${ }^{6,20-22}$ In this study, it was established that telemedicine has a promising and effective role to play in emergency management and clinician-patient interactions. The results indicate how tele-first-aid can benefit the current status of clinical medicine by improving the effectiveness of treatments, bystander first aid, and the clinician-patient relationship, and having positive effects on practice. We share clinicians' insights into how this technology can become part of the existing emergency system and make recommendations for the design of technology based on their perceptions.

All clinicians, clinical specialists, emergency room nurses and doctors, general practitioners, and clinical administrators believed that the growth of tele-first-aid would not replace their clinical work but could be of use in forming treatment plans and providing first-aid care. This is supported by the findings of many other studies on the feasibility and usefulness of telemedicine. ${ }^{23-27}$ The clinicians stated that it would take a long time for people to learn how to use telemedicine systems to seek help and truly depend on such technology. Indeed, the acceptance of this technology will be determined by its appropriate use and its effectiveness in providing timely data to clinicians in both ambulances and hospitals. These factors will be major priorities in stimulating clinicians to adopt telemedicine. 
The clinicians emphasized how this advanced technology can be beneficial for communicating information and emergency treatment instruction and supervision, and many studies have proven the feasibility and usefulness of telemedicine in such applications. ${ }^{23-26}$ The clinicians stated that knowing the patients' vital signs, basic information, and medical history can help optimize emergency treatment plans. ${ }^{27}$ Moreover, firstaid measures must be tailored to the situation since it is necessary to remotely monitor patients in the context of their past medical history. ${ }^{14}$ All participants suggested that they would feel a sense of reassurance if supported by other clinicians, in addition to having the responsibilities for every part of the situation specified by law in advance. The participants recommended that the suggestion and monitoring functions of the technology should be complex rather than simple. This is to ensure that responsibility is delineated, so those acting out of goodwill are protected by a Chinese civil law enacted in March $2017,{ }^{28-30}$ although, in our opinion, this is unfair to patients. The advocated technologies should be made more accurate, quicker, clearer, and safer than they are at present to avoid potential errors. This would be best for everyone. In addition, the instructions provided by such telemedicine systems can be improved as more big data-based technology is developed, allowing clinicians to analyze diagnoses and maximize positive outcomes. Therefore, the cost of recovering from a health event, including medication and hospitalization, can be reduced, benefiting both the healthcare system and patients. These factors are important in encouraging clinicians to adopt telemedicine technologies.

Bystanders can also play a vital role in emergencies by implementing first aid, as noted by many experts. ${ }^{31-33}$ Participants stated that the patients' basic demographic data and health status could be transmitted simultaneously when the first-aid call is initiated. If this is undertaken in the first stage of receiving an emergency client when an ambulance is called and dispatched, it can make a difference to the patient's clinical outcome by increasing the efficiency of collecting patients' data. However, the clinicians mentioned that laypeople's minimal involvement in the prehospital care of an unconscious patient could result in reduced effectiveness of the telemedicine system. In addition, they also doubted how frequently strangers would offer first-aid. Clinicians confirmed that integrating public advice and preferences before designing such systems can improve the rate of layperson assistance, which can notably affect the use and effectiveness of telemedicine in an emergency. Furthermore, the active involvement of laypeople is believed to increase trust between doctors and the public, thus helping improve their relationship. When bystanders perform first aid under the guidance of clinicians via telemedicine, they may be able to help clinicians determine possible changes in the patients' psychology and behaviors. Other professionals support this view. ${ }^{34-38}$

The participants highlighted that emergency treatment in hospitals after a medical rescue is the only option in China. ${ }^{3}$ We thus call for new and multiple approaches in delivering such medical care. Meanwhile, the urgent need for emergency treatment through telemedicine may significantly increase people's enthusiasm for and trust in such technology. However, the development and implementation of tele-first-aid must be embraced by a united population. Participants were inclined to be optimistic in general, suggesting that telemedicine is a blessing rather than a curse, although it will not be a panacea. ${ }^{39,40}$

The COVID-19 outbreak in December 2019 has made it necessary to rethink our current approach to healthcare and how to adapt to emerging healthcare needs in the context of a pandemic. We have learned how to reduce the spread of the virus by implementing social distancing measures, enforcing appropriate mask compliance, and reducing face-to-face contact in a healthy environment. Community transmission of the virus must be prevented to minimize the risk of infection to health professionals. In this regard, necessary telemedicine services could contribute to a strong safeguard of public health. ${ }^{42,43}$

In terms of the medical effect,

the rapid development of telemedicine can save medical costs. However, the risk of misdiagnosis is greater, and statutory provisions are not standardized or universal. This often leads to different standards and coverage offered by service providers.

The duties and responsibilities of the telecom provider, the relationship between the supplier and the patient, the liability and the insurance policy in the event of a medical accident, when there is a system error.

Lack of guidelines for monitoring remote emergency video, guidelines for application development, ethical and legal norms for health informatics professionals and their certification process.

Failure to guarantee wage parity between face-to-face interaction health care providers and telemedicine providers.

In terms of data privacy,

telemedicine includes the extensive use of an electronic format to maintain medical records and record details of 
diagnoses, prescriptions, and follow-up appointments. Electronic medical records can now hold more personal details than any single document. This will amplify issues related to patient privacy, where the scope of data protection varies due to the lack of a common format and the potential for misuse of patient privacy data. Additional concerns about jurisdiction and data ownership become more complex when it comes to cross-border use of telemedicine. Current measures often ignore ethical issues related to professional conduct and relationships, protection of patient autonomy, patient safety, cultural diversity, and human value systems.

"Ethical use of health information."

Differences in the purpose of each medical care operation affect the structure, content, and format of informed consent, resulting in a lack of uniformity in informed consent

In terms of remote devices,

the lack of a strategy to integrate telemedicine regulation into health policy, whether stand-alone medical software can be considered medical devices, the reliability of some symptom checking devices, and the use of these devices may affect the professional relationship between patients and healthcare. Information about the user's health status may increase the level of anxiety among the most sensitive users about whether wearables can be managed as medical devices

and "the high cost of telemedicine implementation due to automation, safety and legality, etc."42,43

This study has limitations. First, from a geographical point of view, the research subjects are mainly medical practitioners from two provinces in central China. Considering the high cost of the hardware, technology, and personnel needs of the remote first aid system, the promotion and acceptance degree of the remote first aid system has a great relationship with the economic level of the region, and people in different regions may have great differences in their attitudes toward the remote first aid system. The central region of China is selected for this study. The economic level of this region is lower than that of the southeastern coastal region, and people's attitude toward the remote emergency system is more conservative. Second, from the point of view of research methods, this research mainly adopts qualitative research methods, collecting data to language and text, so there needs to be an understanding of the language, the language interpretation, and deviations on the text analysis, especially if the interviewee's language is Chinese. The language is translated from Chinese to English, carrying on the analysis. There may be cultural differences, understanding barriers, information omissions, and other problems. Third, from the perspective of sample size, the sample size of this study is 22 , which is not representative enough for potential users of the remote first aid system.

\section{Conclusion}

We studied clinicians' perceptions of the utility of tele-first -aid, its impact on both the doctor-patient relationship and cooperation between organizations, as well as barriers to its implementation. In general, the investigation revealed a positive perception of telemedicine in supporting and facilitating first aid. The acknowledged benefits and limitations of prehospital telemedicine are dramatically impacted by the need for specificity in first-aid scenarios. However, the successful adoption of any technology should be oriented around the preferences of its target users. ${ }^{19,41}$ This study was limited to clinicians in Jiangxi and Henan provinces. In the future, incorporating participants from different geographical areas and other fields of medicine will increase the generalizability of results.

\section{Ethics Approval and Consent to Participate}

This study was conducted with approval from the Ethics Committee of The Second Affiliated Hospital of Nanchang University. This study was conducted in accordance with the declaration of Helsinki. Written informed consent and the participant informed consent included publication of anonymized responses were obtained from all participants.

\section{Funding}

Natural Science Foundation of Jiangxi Province (20192BBG70015), Science and technology project of Jiangxi Health Commission(202130353), Natural Science Foundation of Jiangxi Province(20171BAB215027), Educational Reform Project of Jiangxi Province(JXJG18-1-31).

\section{Disclosure}

The authors reported no conflicts of interest for this work.

\section{References}

1. Su G. Sudden death occurs for a reason. Mod Healthc. 2016;4:18-20.

2. Xu MF, Gu J. Effect evaluation of health education on rescue ability among family members with patients at high risk of sudden cardiovascular. Chin J Health Educat. 2017;33(4):370-373. 
3. Wang LX, Wang FQ. The innovation practice of National CPR popularization and hundreds million of health project. $J$ Chin Res Hosp. 2016;3(4):20-22.

4. Lu CZ, Zhou CW, Zhang YX, et al. The important role of pre-hospital first aid in emergency medicine and medical and health service system. Chin Emerg Med. 2002;06:63-64.

5. Ning J, Liu YJ, Wan YQ. The medical association of remote medical service SWOT analysis [J/OL]. Modern clinical medicine; 2020. Available from: http://www.cnki.com.cn/Article/CJFDTotalYYCD202001021.htm. Accessed July 7, 2021.

6. Adams MK, Date RC, Weng CY. The emergence of telemedicine in retina. Int Ophthalmol Clin. 2016;56(4):47-66. doi:10.1097/ IIO.0000000000000138

7. Wu XQ, Zhang C, Wang LH, et al. Study on remote medical system application in the severe trauma pre-hospital first aid. International Conference on Applied Science; 2017.

8. Sun JL, Wei X, Chen L. Effect of remote first-aid system of "flying rescue of bian que" in pre-hospital first aid for patients with acute chest pain. Sci Med China. 2019;9(05):209-212.

9. Agarwal AK, Gaieski DF, Perman SM, et al. Emergency department based telemedicine: testing the feasibility of using telemedicine as a communication and consultation tool for critically ill patients. Ann Emerg Med. 2013;62(4Suppl):S142-S143. doi:10.1016/j.annemer gmed.2013.07.222

10. Bashford C. POINT OF VIEW. Virtual care: how telemedicine is transforming the delivery of emergency medical services in the field. EMS World. 2010;40(10):31-35.

11. Chung HS, Choa MH, Kim SY, et al. A comprehensive telemedicine system for remote guidance of emergency airway management. $J$ Telemed Telecare. 2007;13(Supp13):29-32. doi:10.1258/1357633 07783247301

12. Taylor P. Evaluating telemedicine systems and services. $J$ Telemed Telecare. 2005;11(4):167-177. doi:10.1258/1357633054068955

13. Megalooikonomou V, Kontos D. Medical data fusion for telemedicine. A model for distributed analysis of medical image data across clinical information repositories. IEEE Eng Med Biol Magazine. 2007;26(5):36-42. doi:10.1109/EMB.2007.901790

14. Wang B, Tang ZR, Wang WB, et al. Application of remote intelligent medical emergency system in pre- hospital first aid. Hosp Admin J Chin People's Liberat Army. 2011;18(11):1007-1009.

15. Castellano N, Gazquez A, García salvador M, et al. Design of a real-time emergency telemedicine system for remote medical diagnosis. Biosyst Eng. 2015;138:23-32. doi:10.1016/j.biosystemseng.2015.03.017

16. Huang W, Li YJ. Effect analysis of emergency trauma treatment based on telemedicine. Chin Health Qual Manag. 2017;24(06):78-81.

17. Yu KQ, Ding J. Application of remote ecg monitoring in emergency treatment of patients with heart disease. J Clin Med. 2017;4 (06):1018-1019.

18. Pan FM, Tang YQ, Lin F, et al. Application effect of remote first-aid system for "flying rescue of bian que" in pre-stroke hospital. China Digit Med. 2019;14(05):103-105.

19. Rogove J, McArthur D, Demaerschalk M, Vespa PM. Barriers to telemedicine: survey of current users in acute care units. Telemedicine and e-Health. 2012;18(1):48-53. doi:10.1089/ tmj.2011.0071

20. Thygerson SM, West JH, Rassbach AR, et al. IPhone apps for first aid: a content analysis. J Consum Health Internet. 2012;16 (2):213-225. doi:10.1080/15398285.2012.673465

21. Shimizu K. Telemedicine by mobile communication. IEEE Eng Med Biol Mag. 1999;18(4):32-44. doi:10.1109/51.775487

22. Carrard VC, Martins MA, Molinabastos CG, et al. WhatsApp: a telemedicine platform for facilitating remote oral medicine consultation and improving clinical examinations-some considerations. Oral Surg Oral Med Oral Pathol Oral Radiol. 2017;123(3):408. doi:10.1016/j.oooo.2016.09.228
23. Fang JL, Collura CA, Johnson RV, et al. Emergency video telemedicine consultation for newborn resuscitations: the mayo clinic experience. Mayo Clinic Proceed. 2016;91(12):1735-1743. doi:10.1016/j.mayocp.2016.08.006

24. Craig J, Patterson V. Introduction to the practice of telemedicine. J Telemed Telecare. 2005;11(1):3-9. doi:10.1177/1357633X0501100102

25. Wang LH. The consider of how does telemedicine promote regional medical informationization consruction? Chongqing Med. 2011;40 (35):3574-3575.

26. Lin QH, Dai Z, Xu YW, et al. The evaluation of the effect of $2 \mathrm{G} / 3 \mathrm{G}$ telemedicine mobile communication system in emergency information transmission in critical patients outside the hospital. Chin J Emerg Resuscitat Disaster Med. 2015;10(11):1025-1028.

27. Wai A, Salib M, Aran S, et al. Patient completion of self-administered medication history forms in the emergency department. Australas Emerg Care. 2019;22(2):103-106. doi:10.1016/j.auec.2019.03.001

28. Gao J, Lin MM, Wang F, et al. "Good man law" supports a good man. People's Weekly. 2017:58-59.

29. Guo MM, Zhang XL, Wei Y. Good man law: the terminator of racketeer. China Newspaper Industry. 2017:90-91.

30. Liu N. The "good man law" in Shanghai tries to solve the problem of rescue. Government Legality. 2017:16-17.

31. He Z, Wynn P, Kendrick D. Non-resuscitative first-aid training for children and laypeople: a systematic review. Emerg Med J EMJ. 2014;31(9):763-768. doi:10.1136/emermed-2013-202389

32. Van de Velde S, Heselmans A, Roex A, et al. Effectiveness of nonresuscitative first aid training in laypersons: a systematic review. Ann Emerg Med. 2009;54(3):1-5. doi:10.1016/j.annemergmed.2008.11.005

33. De Buck E, Van Remoortel H, Dieltjens T, et al. Evidence-based educational pathway for the integration of first aid training in school curricula. Resuscitation. 2015;94:8-22. doi:10.1016/j.resuscitation.2015.06.008

34. Chen PC, Li YC. Using the delphi method to set priorities for health insurance benefit schemes in Taiwan: a policy stakeholder perspective. Taiwan J Public Health. 2015;2:193-203.

35. Xiao LP, Xu JX, Sun H. Investigation and analysis on prehospital emergency medical resources in China. Chin J Disaster Med. 2015;3 (12):662-665.

36. Huo ZH, Ding HS, Gao XC, et al. Establishment and identification of doctor-patient relationship in telemedicine. Chin Hosp Manag. 2017;37(8):27-28.

37. Xie GK. Influence of internet technology on doctor-patient relationships in China. Chin Mental Health J. 2015;10:755-759.

38. Zou ZH, Chen YY, Kong YG, et al. Discussion on the ethical issues and countermeasures of telemedicine. J Med Theory Pract. 2016;29 (15):2137-2139.

39. Meade B, Barnett P. Emergency care in a remote area using interactive video technology: a study in prehospital telemedicine. $J$ Telemed Telecare. 2002;8(2):115-117. doi:10.1258/1357633021937587

40. Leshem-Rubinow E, Assa EB, Shacham Y, et al. Expediting time from symptoms to medical contact utilizing a telemedicine call center. Telemed J E Health. 2015;21(10):801-807. doi:10.1089/ tmj.2014.0227

41. Hartvigsen G, Johansen MA, Hasvold P, et al. Challenges in telemedicine and eHealth: lessons learned from 20 years with telemedicine in Troms? Stud Health Technol Inform. 2007;129:82-86.

42. Nittari G, Khuman R, Baldoni S, et al. Telemedicine practice: review of the current ethical and legal challenges. Telemed $J E$ Health. 2020;26(12):1427-1437. doi:10.1089/tmj.2019.0158

43. Battineni G, Nittari G, Sirignano A, Amenta F. Are telemedicine systems effective healthcare solutions during the COVID-19 pandemic? J Taibah Univ Med Sci. 2021. doi:10.1016/j. jtumed.2021.02.009 


\section{Publish your work in this journal}

The International Journal of General Medicine is an international, peer-reviewed open-access journal that focuses on general and internal medicine, pathogenesis, epidemiology, diagnosis, monitoring and treatment protocols. The journal is characterized by the rapid reporting of reviews, original research and clinical studies

across all disease areas. The manuscript management system is completely online and includes a very quick and fair peer-review system, which is all easy to use. Visit http://www.dovepress.com/ testimonials.php to read real quotes from published authors.

Submit your manuscript here: https://www.dovepress.com/international-journal-of-general-medicine-journal 